\title{
Leaving the Door Open: Trauma, Updating, and the Development of PTSD
}

\section{Symptoms}

Marie R. Sopp ${ }^{1,2^{*}}$, Shilat Haim-Nachum ${ }^{2 *}$, Benedikt E. Wirth ${ }^{1}$, George A. Bonanno ${ }^{3}$, \& Einat Levy-Gigi ${ }^{2,4}$

*These authors contributed equally to this work.

${ }^{1}$ Department of Psychology, Saarland University, Saarbrücken, Germany.

${ }^{2}$ School of Education, Bar-Ilan University, Ramat-Gan, Israel.

${ }^{3}$ Department of Counseling and Clinical Psychology, Teachers College, Columbia University.

${ }^{4}$ The Gonda Multidisciplinary Brain Research Center, Bar-Ilan University, Ramat-Gan, Israel.

\section{Author Note}

Marie R. Sopp: https://orcid.org/0000-0002-9883-5465

Shilat Haim-Nachum: https://orcid.org/0000-0003-4461-5771

Benedikt E. Wirth: https://orcid.org/0000-0001-8039-7316

George A. Bonanno: https://orcid.org/0000-0002-8260-4435

Einat Levy-Gigi: https://orcid.org/0000-0001-5759-6480

Correspondence concerning this article should be addressed to Einat Levy-Gigi. Phone: +972-35318153. Email: einat.levy-gigi@biu.ac.il 


\begin{abstract}
Humans try to make sense of the world using hypotheses that were formed by prior experiences. After trauma, these hypotheses can be exaggerated and resistant to change. This may result in difficulties to update expectations regarding the negative outcomes associated with traumatic stimuli. Critically, it has been proposed that such difficulties may drive the development of posttraumatic stress disorder (PTSD). However, direct evidence on the associations between trauma and impaired expectation updating is still absent. Moreover, it remains unclear whether such an impairment is correlated with PTSD symptoms. To address these gaps, we compared the ability to update traumatic and neutral stimulus-outcome expectations in 81 active-duty firefighters. Participants completed a performance-based updating task and were assessed for PTSD symptoms. We predicted and found a selective impairment in updating trauma-related expectations. This impairment was evident for negativeto-positive but not for positive-to-negative updating. Moreover, impaired negative-to-positive updating was positively associated with PTSD symptoms. These findings support the predictive processing account of PTSD and suggest that strengthening updating processes could be an important goal for promoting resilience after trauma.
\end{abstract}

Keywords: Trauma exposure, updating, predictive processing, PTSD symptoms, high-risk occupations, firefighters. 


\section{Leaving the Door Open: Trauma, Updating, and the Development of PTSD}

\section{Symptoms}

Although many individuals experience potential traumatic events during their lifetime (Kessler et al., 2017), some settings - such as the work environment of first responders (e.g., firefighters, medical personnel, police officers) - inevitably result in high trauma exposure (Kim et al., 2019; Patterson, 2001; Teoh et al., 2019). Critically, first responders are not just exposed to potential trauma once but repeatedly (Geronazzo-Alman et al., 2017), which makes them an ideal population for testing the effects of traumatic exposure as well as possible mechanisms which may account for the development of posttraumatic stress disorder (PTSD). Cognitive models propose that learning processes during trauma may play a key role in PTSD symptom development (for reviews, see Ehlers \& Clark, 2000; Liberzon \& Abelson, 2016). Recent accounts specifically focus on the role of predictive processing (Kim et al., 2020; Kube et al., 2020; Wilkinson et al., 2017). According to these accounts, humans interpret sensory input using hypotheses that are based on prior experiences. By generating prediction errors, new sensory input continuously initiates updating of these hypotheses. However, the likelihood that a prediction error will result in updating depends on the quality of the sensory input. That is, updating is only initiated if the certainty of the sensory information outweighs the confidence (i.e., precision) in the prior hypothesis. Thus, if a prior hypothesis has a very high perceived precision, it can override disconfirmatory information from new sensory input and become highly resistant to change.

Due to the life-threatening nature of traumatic events, trauma-related hypotheses predicting danger are assigned a very high precision (Kube et al., 2020). As a result, subsequent nonthreatening sensory input may fail to update these hypotheses. According to the predictive processing framework of PTSD, this failure may be especially pronounced for new contextual input that trauma-exposed individuals face in their harmless posttrauma environment (Garfinkel et al., 2014). Consequently, trauma-related hypotheses predicting danger become resistant to 
change and are continuously activated in the everyday life of trauma-exposed individuals. By constantly predicting danger in the everyday environment, these hypotheses are believed to promote a sense of continuous threat, which is assumed to facilitate the development of PTSD symptoms. Specifically, the failure to use disconfirmatory contextual information (indicating a harmless environment) for updating is considered critical for PTSD symptom development, since such failure is assumed to decreases the precision of incoming sensory input, giving rise to vivid re-experiencing of trauma in the form of intrusive trauma memories (Kube et al., 2020).

Although the predictive processing framework provides a consistent and timely account of how PTSD symptoms may emerge after trauma exposure, a comprehensive test of its hypotheses is still outstanding. The ultimate goal of the current study was to establish empirical support for the premise of the predictive processing account of PTSD, while further examining the role of updating processes in PTSD etiology. Specifically, we aimed to examine whether updating of stimulus-outcome expectations is reduced for traumatic - as compared to neutral content. Moreover, we sought to test whether reductions are stronger for contextual than for central stimulus features. Finally, we aimed to investigate whether reduced updating is linked to PTSD symptoms.

The first aim was based on the assumption of the predictive processing framework that danger-related hypotheses formed during traumatic events are more resistant to change than hypotheses formed during neutral events. In support of this assumption, Haim-Nachum \& LevyGigi (2019) found that exposure to traumatic as compared to neutral images impaired updating in a subsequent task. In this study, participants were exposed to traumatic or neutral images, and then completed an updating task. In the first phase of the task, neutral stimuli are associated with a negative (i.e., loss) or a positive (i.e., gain) outcome. Following successful acquisition, the associations are reversed and participants need to update the stimulus- outcome associations from positive to negative (i.e., a stimulus associated with gain is now associated with loss) or from negative to positive (i.e., a stimulus associated with loss is now associated with gain). 
Results revealed that participants who had been exposed to traumatic images prior to the task showed reduced updating. This impairment was selective to conditions of negative-to-positive updating, whereas positive-to-negative updating remained intact. A similar pattern was also observed in individuals with repeated trauma exposure as compared to unexposed controls (Levy-Gigi \& Richter-Levin, 2014; Levy-Gigi, Richter-Levin, et al., 2014; Weiss et al., 2019). This may suggest difficulty in learning that stimuli previously predictive of danger are now associated with safety. However, it remains to be shown that traumatic - as compare to neutral - content directly reduces negative-to-positive updating. To test this hypothesis, we assessed updating simultaneously for neutral and traumatic content by directly embedding this different content into the same updating task. Moreover, we used traumatic content that was linked to real-life traumatic events experienced by our sample. We predicted that traumatic as compared to neutral content would impair updating, and that this impairment would be particularly evident in conditions of negative-to-positive updating.

The second aim of the study was based on the assumption of the predictive processing framework that the extent to which trauma-related hypotheses are resistant to updating should be directly linked to PTSD symptoms. That is, trauma-exposed individuals with greater resistance to updating of trauma-related hypotheses should show more severe PTSD symptoms than those with lower resistance. Consistent with this assumption, previous studies demonstrate that individuals with repeated trauma exposure experience more PTSD symptoms if they show rigid regulation rather than flexible regulation (Levy-Gigi et al., 2016; Rodin et al., 2017). However, in the context of updating processes, it remains to be examined whether impaired negative-to-positive updating of traumatic content correlates with PTSD symptoms. Correspondingly, we hypothesized to find a significant association between impaired negativeto-positive updating for traumatic content and PTSD symptom severity.

Finally, tying together both aims, the predictive processing account posits that traumaexposed individuals particularly struggle to use contextual information to update trauma-related 
hypotheses and that this specific deficit in context updating is linked to PTSD symptoms. To investigate such effects, we used an updating task that differentiates between updating target (presented at central vision) and contextual (presented peripherally) stimulus features, enabling the independent assessment of both target and context updating. Previous studies that utilized this task with neutral stimulus-outcome associations revealed selective impairments in context updating in trauma-exposed individuals. However, no specific associations were found between this updating deficit and level of PTSD symptoms (Levy-Gigi \& Richter-Levin, 2014; LevyGigi et al., 2015). Here, we tested whether using traumatic content would lead to similar impairments in negative-to-positive context updating and whether this selective impairment correlates with PTSD symptoms.

In sum, to address each of these aims, the current study used a novel variant of the updating task as described above with a sample of firefighters. In this variation, targets were presented with context images that were either neutral or traumatic followed by positive (i.e., gain) or negative (i.e., loss) outcomes. Traumatic content was selected to resemble traumatic scenes that first responders may witness in their work environment. By using this task in a sample of firefighters, we were able to track whether participants were equally successful in updating neutral content and traumatic content related to their work environment. After the task, firefighters completed a self-report questionnaire assessing PTSD symptoms of the past month.

\section{Methods}

\section{Participants}

Eighty-two Israeli male firefighters participated in the study. Sample size was based on the detection of medium-sized associations ( $r=.30$; two sided) between updating and PTSD symptoms with a power of 0.80 . One participant was discarded because he did not reach the required acquisition criterion (i.e., six consecutive correct responses during acquisition; see 2.2.1 for details). Hence, the final analysis sample comprised 81 participants $\left(M_{\text {age }}=29.02\right.$, 
$\left.S D_{\text {age }}=4.99\right)$. All participants had completed military service, with 40 participants indicating that they had been involved in combat and experienced military-related events.

Participants were recruited while taking part in a basic training course. Assessment took place after they had completed the 8-month course. During this time, they were involved in active service, which had included exposure to traumatic events (for an overview of events that this population regularly experiences see Levy-Gigi et al., 2016). A team of researchers introduced all participating firefighters to the study objectives and procedures. Those volunteering to participate were asked to provide written informed consent and completed the updating task as well as several self-report questionnaires. Participation was restricted to individuals who did not report a current or past diagnosis of Axis I disorders other than PTSD, suicidal ideation, substance abuse within the past month, or neurological injuries (e.g., concussion) or diseases (e.g., epilepsy). All study procedures were approved by the local ethics committees.

\section{Measures}

\section{Assessment of updating for traumatic and neutral content}

Updating for traumatic and neutral content was assessed using a novel task, which was based on a validated updating task using neutral stimuli (Haim-Nachum \& Levy-Gigi, 2019, 2021; Levy-Gigi \& Richter-Levin, 2014; Levy-Gigi et al., 2014; 2015; Zabag et al., 2020). During the acquisition phase, participants see the front view of a wall with a door (see Figure 1). The wall serves as the context and displays either a neutral or traumatic image. The door is white and contains a symbol that is prominently displayed in the center, serving as the target. After the presentation of each door, participants are asked whether they wish to open the door or not. After opening the door, participants either receive a reward or lose a portion of their previous rewards. Thus, participants learn by trial and error to predict the outcome of each door according to its surrounding wall and symbol. Participants see a total of four doors during this phase. Two doors are surrounded by neutral images and two doors are surrounded by traumatic 
images. One door of each stimulus category is associated with a positive outcome (gain) whereas the other one is associated with a negative outcome (loss). Outcomes of individual doors are counterbalanced across participants. To complete the acquisition phase and move on to the retention and updating phase, participants need to reach a criterion of six consecutive trials with correctly predicted outcomes within a minimum of 40 trials. Correct predictions are reflected in opening doors that are associated with positive outcomes and not opening those that are associated with negative outcomes. Participants who do not reach this criterion have a chance to complete a maximum of 16 cycles of 8 trials each. If they reach the criterion after one cycle, they move on to the retention and updating phase. If they do not reach the criterion in all 16 cycles, the experiment is terminated prematurely.

--Insert Figure 1 around here--

After successful completion of the acquisition phase, participants enter the retention and updating phase. In this phase, participants see the original doors (associated with either positive or negative outcome) as well as new doors. New doors share either the context or target of the original doors but are associated with the opposite outcome. During context updating trials, the same background picture is presented with a new symbol. During target updating trials, the same symbol is presented with a new background picture. Thus, successful responding to new doors requires updating the association rule of either the original target or the original context from positive to negative or from negative to positive. The entire retention and updating phase consists of 120 trials (10 trials per condition). Trials are presented blockwise (one block consisting of one trial per condition) with a random order of trials within blocks. Performance is quantified by calculating accuracy rates for each condition.

\section{Assessment of PTSD symptoms}

The PTSD Checklist for DSM-IV (PCL) was used to assess PTSD symptoms during the past month in accordance with DSM-IV symptom criteria (Weathers et al., 1993). The questionnaire comprises 17 items, which are rated on a scale ranging from 1 (= not at all) to 5 
(= extremely). Reliability and validity of the original measure are good and internal consistency is high (Blanchard et al., 1996). The total score across symptom domains was used for the current analyses. The PCL score can range between 17 and 85 . Scores in the current sample ranged from 17 to 35. Since PCL scores were non-normally (right skewed) distributed, they were log-transformed to approximate normality. Transformed scores were used for all analyses.

\section{Assessment of covariates}

Exposure to adverse life events was assessed using a 10-item checklist. The checklist includes common adverse events such as death of a parent/sibling, chronic illness in the family, severe economic difficulties, and domestic violence. Participants were asked to indicate whether the event happened to them (Yes/No/I do not remember) and whether it happened during childhood (age $<10$ ), adolescence $(18>$ age $>10)$ and/or adulthood (age $>18)$. A sum score of experienced events was computed separately for each life period for further analyses. Participants reported a range of 0 to 3 experiences during childhood, 0 to 3 experiences during adolescence, and 0 to 8 experiences during adulthood.

Depression was assessed using the Beck Depression Inventory (BDI-II) developed by Beck et al. (1996). High reliability and validity of the scale have been established by previous research (Whisman et al., 2000; for review, see Wang \& Gorenstein, 2013). Each item is measured on a scale from 0 to 3, with total scores ranging from 0 to 63; higher scores indicate greater levels of depression. The sum score of all items was used for the current analyses. BDI scores ranged from 0 to 19 in the current sample.

Verbal intelligence was assessed using the Wechsler Adult Intelligence Scale-III (WAISIII) Vocabulary subtest (Wechsler, 1997). The test requires participants to try to define up to 30 words. The reliability and validity of the test has been extensively documented and also extends to clinical populations (Ryan \& Rosenberg, 1984).

\section{Data analysis}


Data analysis was performed using IBM SPSS Statistics 25. The alpha level was set to .05 for all analyses. Our first set of analyses investigated the impact of traumatic content on updating. We conducted an ANOVA including the factors Stimulus Type (trauma vs. neutral), Valence (negative-to-positive vs. positive-to-negative reversal), and Updating Type (target vs. context). Significant interaction effects were followed up by conducting independent $t$-tests. Partial $\eta^{2}$ was calculated to illustrate effect sizes.

Our second set of analyses aimed to investigate the association between negative-to-positive context updating and PTSD symptoms. To this end, we conducted hierarchical linear regression analyses. In a first step, all control variables (Exposure to adverse life events, depression, and verbal intelligence) and acquisition performance were simultaneously entered into the model to account for any variance explained by these variables. In a second step, negative-to-positive updating was entered as predictor. Analyses were conducted separately for neutral and traumatic content.

If the model including negative-to-positive updating as predictor yielded a significant improvement of model prediction, we conducted a separate analysis entering both context and target updating as independent predictors to examine incremental validity. In order to test the selectivity of found effects, analyses were repeated for positive-to-negative updating (see Supplementary file A for details). Standardized regression coefficients $(\beta)$ with t-values as well as overall model tests are reported. Effect sizes are illustrated in terms of the amount of variance accounted for by each model (adjusted $\mathrm{R}^{2}$ ).

\section{Results}

\section{Impact of traumatic vs. neutral content on updating.}

An ANOVA including the factors Stimulus Type (neutral vs. traumatic), Valence (positiveto-negative vs. negative-to-positive reversal), and Updating Type (target vs. context updating) and accuracy rates as dependent variable revealed a significant main effect of Updating Type, 
$F(1,80)=15.80, p<.001, \eta_{p}^{2}=.165$, reflecting higher accuracy rates for context updating as opposed to target updating, $t(80)=3.97, p<.001$. In addition, a significant interaction between Stimulus Type and Valence emerged, $F(1,80)=10.48, p=.002, \eta_{p}^{2}=.116$ (see Figure 2). For negative-to-positive updating, lower accuracy rates were evident for traumatic as compared to neutral stimuli, $t(80)=2.89, p=.005$. Conversely, no differences were evident for positive-tonegative updating, $t(80)=1.76, p=.083$. Finally, a significant interaction between Stimulus Type and Updating Type, $F(1,80)=4.32, p=.041, \eta_{p}{ }^{2}=.051$ emerged (see Figure 3). Followup analyses revealed that accuracy rates were lower for traumatic than for neutral target updating, $t(80)=3.21, p=.002$. By contrast, no significant difference emerged for context updating, $t(80)=0.60, p=.553$. None of the other main or interaction effects reached significance $(p>.280)$. In summary, our analyses indicate that traumatic content selectively weakened negative-to-positive rather than positive-to-negative updating and target rather than context updating.

--Insert Figure 2 and Figure 3 around here--

\section{Associations between updating and PTSD symptoms.}

--Insert Table 1 around here--

Bivariate correlations between updating, PTSD symptoms, and covariates are presented in Table 1. In order to assess associations between updating and PTSD symptoms while controlling for acquisition performance, depression, verbal intelligence, and exposure to adverse life events, we conducted a series of hierarchical regression analyses (see Table 2 and 3). Including negative-to-positive updating for traumatic content as independent variable significantly improved prediction of PTSD symptoms as compared to the baseline model, $\Delta R^{2}$ $=.047, F(1,72)=5.56, p=.021$. That is, greater negative-to-positive updating for traumatic stimuli was associated with fewer PTSD symptoms (see Table 2). Introducing negative-to- 
positive updating for neutral content as independent variable did not significantly improve prediction of PTSD symptoms as compared to the baseline model, $\Delta R^{2}=.021, F(1,72)=2.31$, $p=.133$ (see Table 3). Similarly, positive-to-negative updating for neutral and traumatic content did not significantly improve prediction (all ps > .491; see Supplementary file A).

--Insert Table 2 around here-

In order to assess whether the association between negative-to-positive updating for traumatic content and PTSD symptoms is stronger for target or context updating, we simultaneously introduced negative-to-positive target and context updating into the baseline model. The analysis yielded a significant improvement in prediction, $\Delta R^{2}=.064, F(2,71)=$ $3.81, p=.027$. Inspection of individual regression weights revealed that reduced target updating predicted PTSD symptoms whereas reduced context updating did not (see Table 2).

\section{Discussion}

The current study investigated whether traumatic - as compared to neutral - content impairs updating and whether this impairment correlates with PTSD symptoms in a sample of activeduty firefighters. In line with the predictive processing framework, we postulated and found that traumatic content impaired updating compared to neutral content. This effect was only evident for negative-to-positive updating and not for positive-to-negative updating. Moreover, we found that impaired negative-to-positive updating selectively predicted PTSD symptoms of the past month. Finally, in contrast to our hypotheses, we found that traumatic - as compared to neutral content - impairs target rather than context updating. Correspondingly, only impaired negative-to-positive target - and not context - updating for traumatic content predicted PTSD symptoms.

Our finding concurs with previous research showing that negative-to-positive updating is selectively impaired in trauma-exposed individuals (Levy-Gigi \& Richter-Levin, 2014; LevyGigi, Richter-Levin, et al., 2014; Radell et al., 2017; Zabag et al., 2020). Most importantly, our 
study extends previous research in two important ways: Firstly, by contrasting updating performance for neutral and traumatic content, we provide direct support for the assumption that traumatic content is more resistant to updating compared to neutral content. Secondly, our study is the first to demonstrate a link between reduced updating and PTSD symptoms. This is especially interesting since previous studies demonstrated impaired updating of neutral associations in individuals with repeated traumatic exposure compared to unexposed controls (Levy-Gigi \& Richter-Levin, 2014; Levy-Gigi, Richter-Levin, et al., 2014). However, these general updating impairments did not correlate with PTSD symptoms, and hence were considered a hidden price of repeated trauma. The current findings suggest that using traumatic content may capture a direct explicit price of repeated traumatic exposure, demonstrated in the level of PTSD symptoms.

On a conceptual level, our findings are strongly aligned with the premise of the predictive processing account. Nevertheless, we found a deviation: Traumatic content affected target rather than context updating. Moreover, target rather than context updating was correlated with PTSD symptoms. This finding appears to be at odds with the assumption of the predictive processing framework that PTSD symptoms specifically emerge because trauma-exposed individuals fail to use disconfirmatory contextual information in their environment to update trauma-related hypotheses (Kube et al., 2020). Moreover, these results contradict previous studies (Haim-Nachum \& Levy-Gigi, 2021; Levy-Gigi et al., 2015) showing that context - and not target - updating is specifically impaired in trauma-exposed individuals with PTSD symptoms. A possible explanation for this inconsistency is that previous studies and the current one differed in sample composition. Whereas previous studies examined first responder samples with a wide range of traumatic exposure due to high variance in years of work experience (Levy-Gigi, Richter-Levin, et al., 2014), the current study investigated a homogenous sample of firefighters after an 8-month period of trauma exposure. Hence, effects in our sample may only reflect early responses to work-related trauma whereas effects in 
previous samples may also reflect long-term adjustment to chronic work-related trauma (Lee et al., 2020). Future research should further investigate this hypothesis by contrasting subsamples with short-term and chronic work-related trauma exposure to assess how this potential moderators influences context and target updating.

Alternatively, it is possible that differences between the current task and the task used by previous studies may account for the fact that we found effects for target rather than context updating. That is, previous tasks used a highly salient context feature (i.e., background color) whereas the current task used complex scenes, which may have altered processing demands and subsequent context updating. Moreover, it is important to note that our task varied neutral and traumatic context pictures, whereas targets were always neutral. Hence, the task features a certain imbalance since traumatic content was only presented as context and never as target. Importantly, such imbalance nicely imitates real post traumatic situations in which neutral and trauma-related contents typically co-occur. However, it is possible that in these situations traumatic context images trigger attentional avoidance (Mackintosh \& Mathews, 2003; Sagliano et al., 2021), which in turn may affect the ability to learn that the same old targets can predict positive outcomes when presented in new contexts, similar to situations in which the same sound of airplanes in different battle fields may rather signal a negative outcome (enemy attack) or a positive outcome (rescue forces). Moreover, since attentional avoidance has been shown to be greater in individuals with PTSD symptoms (Schoorl et al., 2014), this impairment may have been more pronounced in symptomatic individuals. Future studies may aim to include traumatic images of both target and context and investigate their differential impact on updating.

Finally, the lack of alignment between our selective finding for target rather than context updating and the assumption of the predictive processing framework concerning contextual processing may be related to discrepancies between different definitions of context. Specifically, in the task which was utilized in our study, both targets (i.e., symbol on a door) 
and contexts (i.e., wall illustration) are processed as parts of a complex scene configuration. According to the predictive updating framework, these components could thus be considered equally contextual since they are scene details that are processed incidentally without any specific instruction that guides attention (Kube et al., 2020). Hence, if transferred to real-life conditions, targets and contexts could reflect cues of a harmless post trauma environment used to update trauma-related hypotheses. Future research may aim to further test the relationship between target and context updating by using top-down definitions, in which explicit instructions to focus attention on one of the two elements are given.

Although our results provide important insights, several limitations should be considered. First, presenting traumatic pictures rather than examining updating related to real-life trauma limits generalization of our findings. However, it is difficult to examine fine-grained processes such as updating during or immediately after real-life trauma. For this reason, using materials that are conceptually related to individual trauma is considered a valid approach to investigate peri- and posttraumatic processes (e.g., Ehring et al., 2011). Secondly, it is important to note that the majority of participants in this study reported subclinical PTSD symptoms. However, investigating samples with a wide range of subclinical symptoms aligns with recent views that promote characterizing mental disorders in terms of varying degrees of dysfunction in general psychological and biological systems rather than limiting observation to dichotomous clinical definitions (e.g., Carcone et al., 2017; Cuthbert et al., 2013; Insel et al., 2010). A further limitation is that our design did not include a non-exposed control group. As a result, we were not able to investigate trauma-related alterations in neutral updating but only alterations in traumatic as compared to neutral updating. Thus, we were not able to directly replicate analyses of previous studies finding reduced neutral updating in trauma-exposed samples (Levy-Gigi \& Richter-Levin, 2014; Levy-Gigi, Richter-Levin, et al., 2014; Weiss et al., 2019). Nevertheless, it should be noted that since neutral updating performance did not approach ceiling levels, it is possible that such a reduction also exists in the current sample. Finally, our sample comprised 
only of male participants that were exposed to occupational trauma, constituting a relatively homogenous population. However, since trauma exposure is pervasive in several settings, our findings may generalize to other populations of first responders (e.g., police officers, medical personnel) and also beyond (e.g., civilians living in war zones, children growing up in abusive families).

Despite these limitations, the current findings contribute to the current literature. On the one hand, they support the premise of the predictive processing account of PTSD (Kube et al., 2020) that traumatic stress impacts subsequent updating of trauma-related hypotheses, which is in turn linked to PTSD symptom development. On the other hand, they underline the clinical relevance of updating processes in the context of PTSD. That is, if replicated, our findings indicate that helping traumatized individuals to acquire and maintain adaptive updating patterns may be a promising avenue for promoting resilience after trauma. This view is supported by research showing that cognitive training early after trauma exposure reduces subsequent symptom development (Ben-Zion et al., 2018). Moreover, in symptomatic individuals, training of updating processes may help to reduce existing symptoms. Initial studies examining the effects of cognitive training have shown promising effects both as a standalone treatment (Bomyea et al., 2015; Larson et al., 2019) and as adjunctive treatment for PTSD (Crocker et al., 2019). Critically, to the best of our knowledge, no study to date has trained updating of outcome expectations in trauma-exposed individuals to assess effects on PTSD symptoms. Based on the current findings, such research appears both timely and warranted. 


\section{References}

Beck, A. T., Steer, R. A., \& Brown, G. (1996). Beck depression inventory-II. Psychological Assessment.

Ben-Zion, Z., Fine, N. B., Keynan, N. J., Admon, R., Green, N., Halevi, M., ... \& Shalev, A. Y. (2018). Cognitive flexibility predicts PTSD symptoms: observational and interventional studies. Frontiers in psychiatry, 9, 477.

Blanchard, E. B., Jones-Alexander, J., Buckley, T. C., \& Forneris, C. A. (1996). Psychometric properties of the PTSD Checklist (PCL). Behaviour Research and Therapy, 34(8), 669673.

Bomyea, J., Stein, M. B., \& Lang, A. J. (2015). Interference control training for PTSD: A randomized controlled trial of a novel computer-based intervention. Journal of anxiety disorders, 34, 33-42.

Carcone, D., \& Ruocco, A. C. (2017). Six years of research on the national institute of mental health's research domain criteria (RDoC) initiative: a systematic review. Frontiers in Cellular Neuroscience, 11, 46.

Crocker, L. D., Jurick, S. M., Thomas, K. R., Keller, A. V., Sanderson-Cimino, M., Boyd, B., ... \& Jak, A. J. (2018). Worse baseline executive functioning is associated with dropout and poorer response to trauma-focused treatment for veterans with PTSD and comorbid traumatic brain injury. Behaviour research and therapy, 108, 68-77.

Cuthbert, B. N., \& Kozak, M. J. (2013). " Constructing constructs for psychopathology: The NIMH research domain criteria": Correction to Cuthbert and Kozak (2013). 
Ehlers, A., \& Clark, D. M. (2000). A cognitive model of posttraumatic stress disorder. Behaviour Research and Therapy, 38(4), 319-345.

Ehring, T., Kleim, B., \& Ehlers, A. (2011). Combining clinical studies and analogue experiments to investigate cognitive mechanisms in posttraumatic stress disorder. International Journal of Cognitive Therapy, 4(2), 165-177.

Garfinkel, S. N., Abelson, J. L., King, A. P., Sripada, R. K., Wang, X., Gaines, L. M., \& Liberzon, I. (2014). Impaired contextual modulation of memories in PTSD: an fMRI and psychophysiological study of extinction retention and fear renewal. Journal of Neuroscience, 34(40), 13435-13443.

Geronazzo-Alman, L., Eisenberg, R., Shen, S., Duarte, C. S., Musa, G. J., Wicks, J., ... \& Hoven, C. W. (2017). Cumulative exposure to work-related traumatic events and current post-traumatic stress disorder in New York City's first responders. Comprehensive psychiatry, 74, 134-143.

Haim-Nachum, S., \& Levy-Gigi, E. (2019). A chink in the armor: The influence of training on generalization learning impairments after viewing traumatic stimuli. Cognition, 193, 104021.

Haim-Nachum, S., \& Levy-Gigi, E. (2021). To Be or Not to Be Flexible: Selective impairments as a means to differentiate between depression and PTSD symptoms. Journal of Psychiatric Research, 136, 366-373.

Insel, T., Cuthbert, B., Garvey, M., Heinssen, R., Pine, D. S., Quinn, K., ... \& Wang, P. (2010). Research domain criteria (RDoC): toward a new classification framework for research on mental disorders. The American journal of psychiatry, 167(7), 748-751. 
Kessler, R. C., Aguilar-Gaxiola, S., Alonso, J., Benjet, C., Bromet, E. J., Cardoso, G., Degenhardt, L., de Girolamo, G., Dinolova, R. V., Ferry, F., Florescu, S., Gureje, O., Haro, J. M., Huang, Y., Karam, E. G., Kawakami, N., Lee, S., Lepine, J.-P., Levinson, D., Navarro-Mateu, F., Pennell, B.-E., Piazza, M., Posada-Villa, J., Scott, K. M., Stein, D. J., Ten Have, M., Torres, Y., Viana, M. C., Petukhova, M. V., Sampson, N. A., Zaslavsky, A. M., \& Koenen, K. C. (2017). Trauma and PTSD in the WHO World Mental Health Surveys. European Journal of Psychotraumatology, 8(sup5), 1353383.

Kim, M. J., Jeong, Y., Choi, Y. S., Seo, A. R., Ha, Y., Seo, M., \& Park, K. S. (2019). The association of the exposure to work-related traumatic events and work limitations among firefighters: A cross-sectional study. International Journal of Environmental Research and Public Health, 16(5), 756.

Kim, M., Park, B., \& Young, L. (2020). The psychology of motivated versus rational impression updating. Trends in Cognitive Sciences, 24(2), 101-111.

Kube, T., Berg, M., Kleim, B., \& Herzog, P. (2020). Rethinking post-traumatic stress disorderA predictive processing perspective. Neuroscience \& Biobehavioral Reviews, 113, 448460.

Levy-Gigi, E., Bonanno, G. A., Shapiro, A. R., Richter-Levin, G., Kéri, S., \& Sheppes, G. (2016). Emotion regulatory flexibility sheds light on the elusive relationship between repeated traumatic exposure and posttraumatic stress disorder symptoms. Clinical Psychological Science, 4(1), 28-39.

Levy-Gigi, E., \& Richter-Levin, G. (2014). The hidden price of repeated traumatic exposure. Stress, 17(4), 343-351. 
Levy-Gigi, E., Richter-Levin, G., \& Kéri, S. (2014). The hidden price of repeated traumatic exposure: different cognitive deficits in different first-responders. Frontiers in Behavioral Neuroscience, 8, 281.

Levy-Gigi, E., Szabo, C., Richter-Levin, G., \& Kéri, S. (2015). Reduced hippocampal volume is associated with overgeneralization of negative context in individuals with PTSD. Neuropsychology, 29(1), 151.

Liberzon, I., \& Abelson, J. L. (2016). Context processing and the neurobiology of posttraumatic stress disorder. Neuron, 92(1), 14-30.

Mackintosh, B., \& Mathews, A. (2003). Don't look now: Attentional avoidance of emotionally valenced cues. Cognition and Emotion, 17(4), 623-646.

Radell, M. L., Beck, K. D., Gilbertson, M. W., \& Myers, C. E. (2017). Post-traumatic stress disorder symptom burden and gender each affect generalization in a reward-and punishment-learning task. PLoS ONE, 12(2), e0172144.

Rodin, R., Bonanno, G. A., Rahman, N., Kouri, N. A., Bryant, R. A., Marmar, C. R., \& Brown, A. D. (2017). Expressive flexibility in combat veterans with posttraumatic stress disorder and depression. Journal of affective disorders, 207, 236-241.

Ryan, J. J., \& Rosenberg, S. J. (1984). Validity of the verbal IQ as a short form of the Wechsler adult intelligence scale-revised. Journal of clinical psychology, 40(1), 306-308.

Sagliano, L., Conson, M., Saporito, G., Carolei, A., Sacco, S., \& Pistoia, F. (2021). "Far from the mind": Preliminary evidence of avoidance bias for emotional facial expressions among earthquake victims. International Journal of Disaster Risk Reduction, 59, 102273. 
Schoorl, M., Putman, P., Van Der Werff, S., \& Van Der Does, A. W. (2014). Attentional bias and attentional control in posttraumatic stress disorder. Journal of Anxiety Disorders, 28(2), 203-210.

Shrira, A., Shmotkin, D., \& Litwin, H. (2012). Potentially traumatic events at different points in the life span and mental health: findings from SHARE-Israel. American Journal of Orthopsychiatry, 82(2), 251.

Teoh, K. R. H., Lima, E., Vasconcelos, A., Nascimento, E., \& Cox, T. (2019). Trauma and work factors as predictors of firefighters' psychiatric distress. Occupational Medicine, 69(8-9), 598-603.

Wang, Y. P., \& Gorenstein, C. (2013). Psychometric properties of the Beck Depression Inventory-II: a comprehensive review. Brazilian Journal of Psychiatry, 35(4), 416-431.

Weathers, F. W., Litz, B. T., Herman, D. S., Huska, J. A., \& Keane, T. M. (1993). The PTSD Checklist (PCL): Reliability, validity, and diagnostic utility. annual convention of the international society for traumatic stress studies, San Antonio, TX,

Weiss, O., Levy-Gigi, E., Adelson, M., \& Peles, E. (2019). Methadone maintenance treatment patients with a history of childhood trauma succeed more in a cognitive paradigm that is associated with a negative reward. Psychiatry research, 271, 381-388.

Whisman, M. A., Perez, J. E., \& Ramel, W. (2000). Factor structure of the Beck Depression Inventory-Second Edition (BDI-ii) in a student sample. Journal of Clinical Psychology, 56(4), 545-551. 
Zabag, R., Deri, O., Gilboa-Schechtman, E., Richter-Levin, G., \& Levy-Gigi, E. (2020). Cognitive flexibility in PTSD individuals following nature adventure intervention: is it really that good? Stress, 23(1), 97-104. 


\section{Figure captions}

Figure 1. Illustration of the updating task. Traumatic pictures are blurred due to sensitive content.

Figure 2. Illustration of the interaction between Updating Type and Stimulus Type. Error bars depict \pm 1 SEM (standard error of the mean). Points represent individual participants. The graphs were made using the ggplot2 library (Wickham, 2016) for R (R Core Team, 2019).

Figure 3. Illustration of the interaction between Valence and Stimulus Type. Error bars depict \pm 1 SEM (standard error of the mean). Points represent individual participants. The graphs were made using the ggplot2 library (Wickham, 2016) for R (R Core Team, 2019). 


\section{Acquisition}

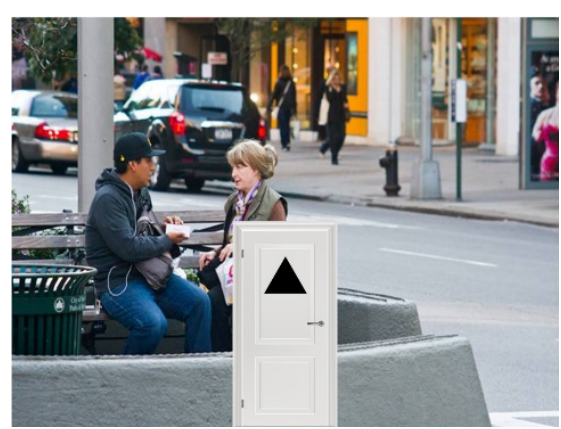

(ن)

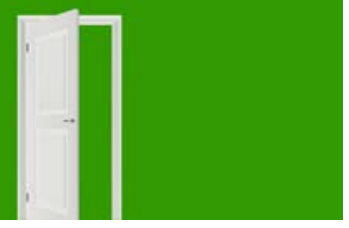

Neutral

Positive
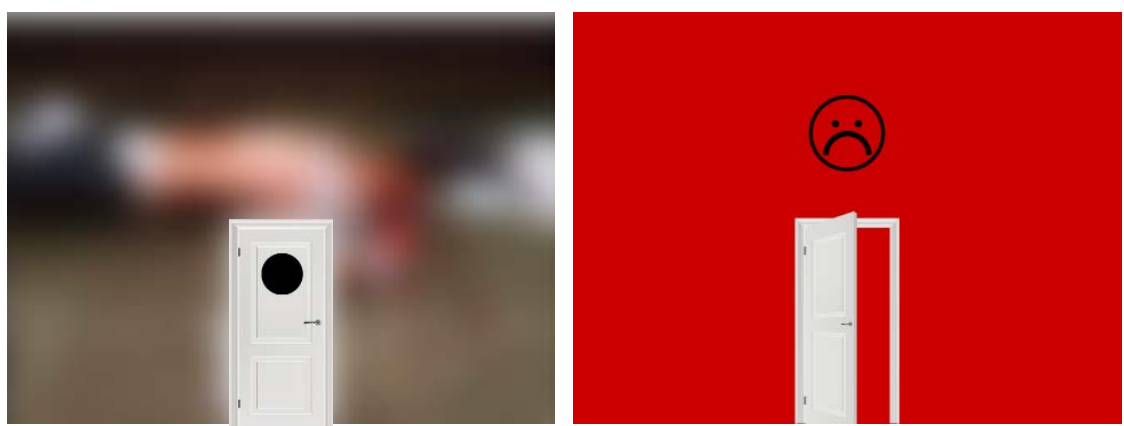

Traumatic

Negative

\section{Updating}
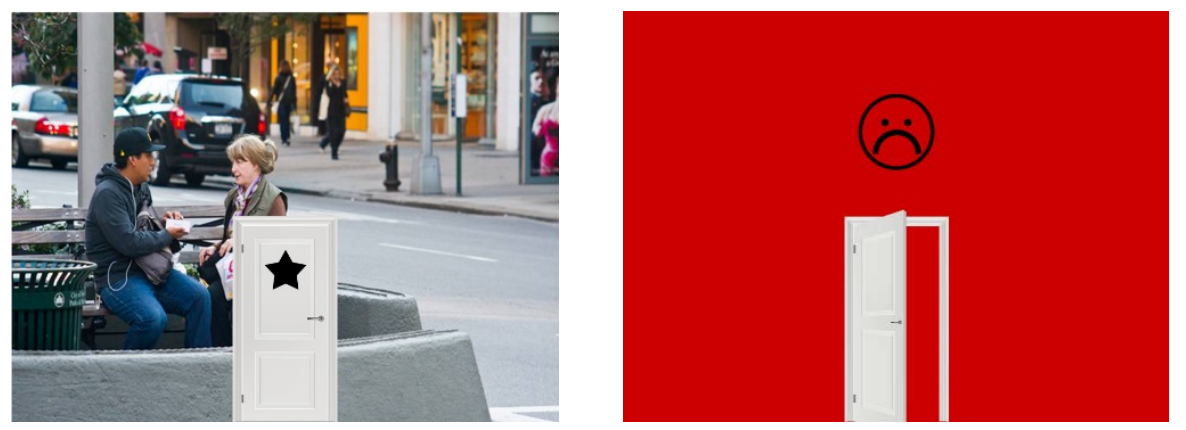

Positive-tonegative context updating for neutral stimuli
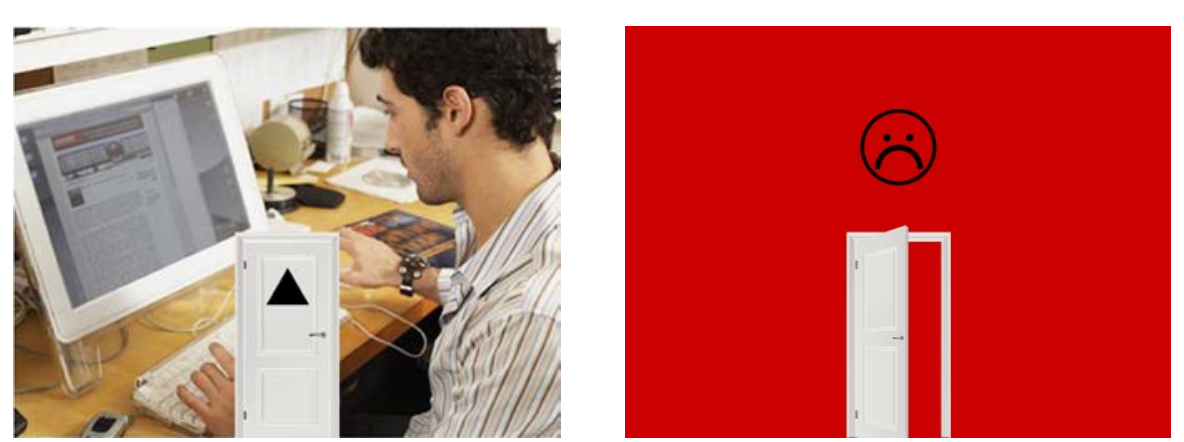

Positive-tonegative target updating for neutral stimuli
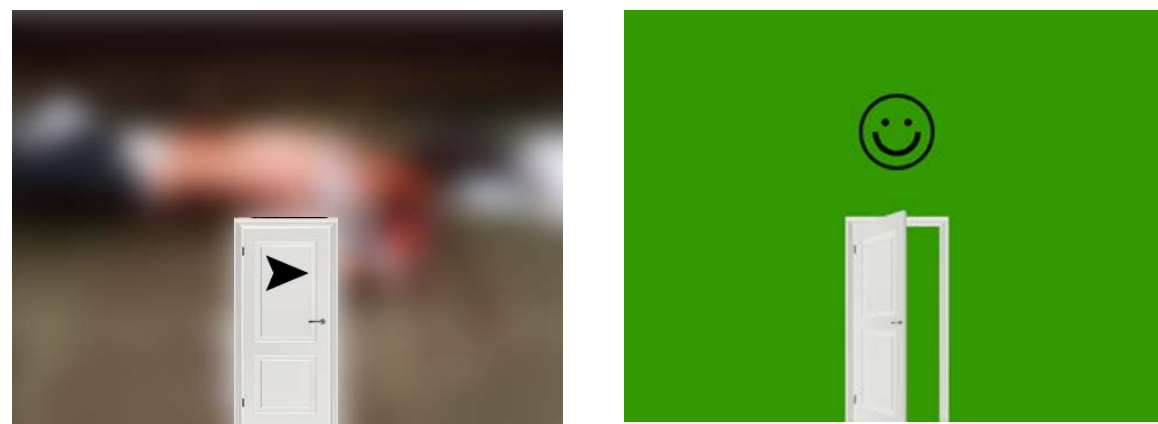

Negative-topositive context updating for traumatic stimuli
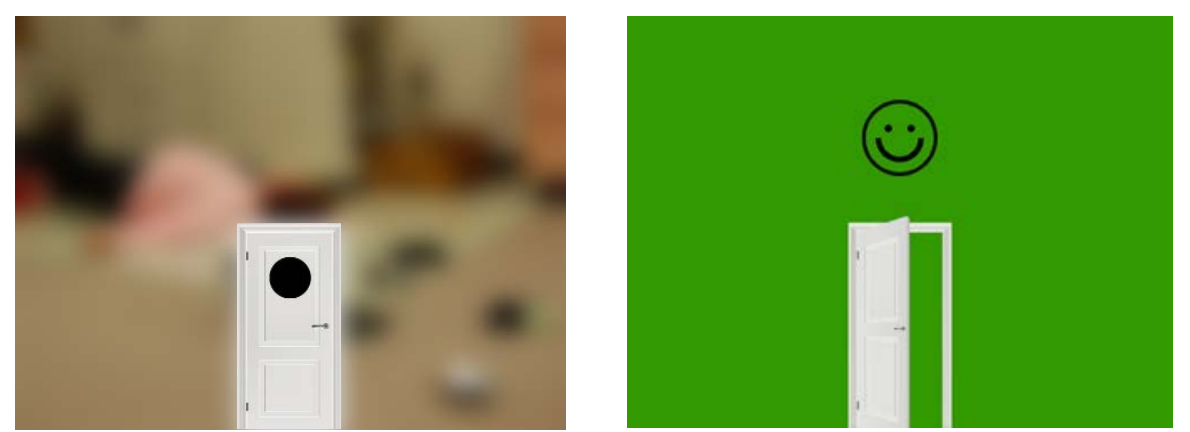

Negative-topositive target updating for traumatic stimuli 


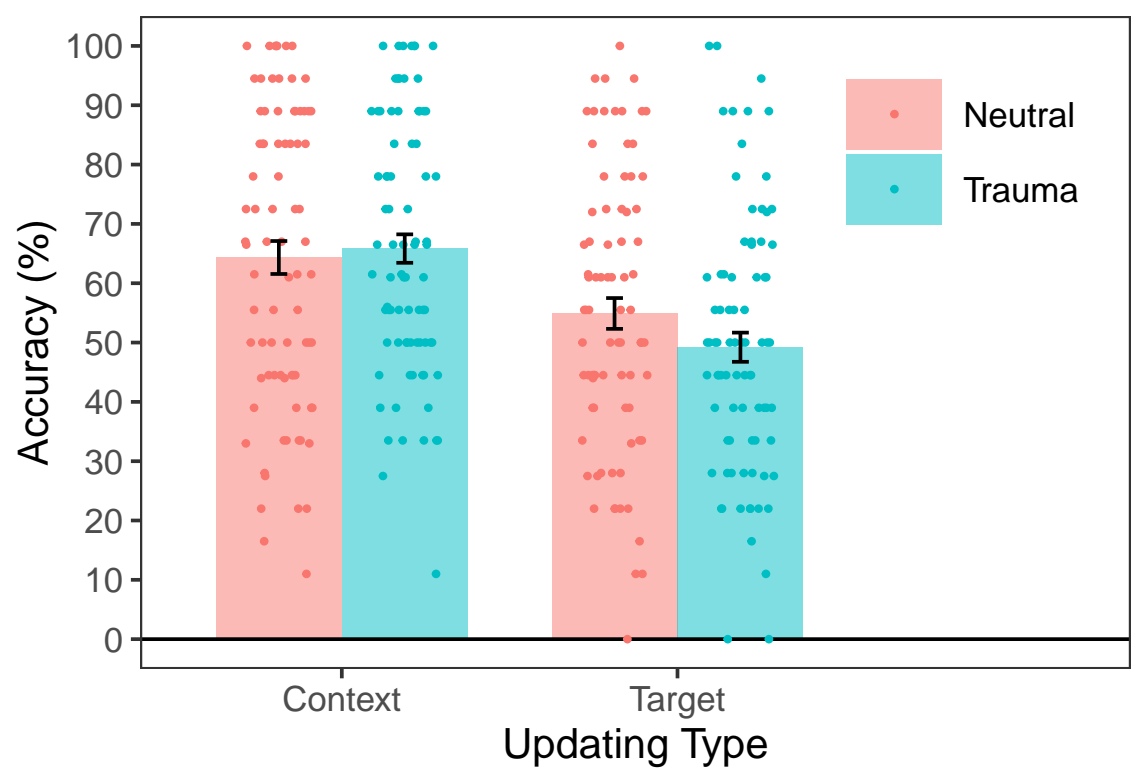


Table 1. Bivariate associations between updating, PTSD symptoms, and covariates.

\begin{tabular}{|c|c|c|c|c|c|c|c|c|c|c|c|c|}
\hline Measures & 1 & 2 & 3 & 4 & 5 & 6 & 7 & 8 & 9 & 10 & 11 & 12 \\
\hline 1. N-to-P updating trauma & - & $.45^{* *}$ & $.78^{* *}$ & $.73 * *$ & $.22^{*}$ & $.41^{* *}$ & $-.23 *$ & -.09 & .16 & .00 & -.15 & .07 \\
\hline 2. N-to-P updating neutral & - & - & $.51^{* *}$ & .16 & $.71 * *$ & $.65^{* *}$ & -.11 & .03 & .11 & .10 & .03 & .05 \\
\hline 3. Target $\mathrm{N}$-to-P updating trauma & - & - & - & .14 & $.51 * *$ & .17 & $-.23 *$ & -.19 & .12 & -.05 & -.10 & .02 \\
\hline 4. Context N-to-P updating trauma & - & - & - & - & -.21 & $.45^{* *}$ & -.11 & .07 & .13 & .06 & -.12 & .08 \\
\hline 5. Target $N$-to-P updating neutral & - & - & - & - & - & -.07 & -.08 & -.04 & .19 & .16 & .05 & -.07 \\
\hline 6. Context $\mathrm{N}$-to-P updating neutral & - & - & - & - & - & - & -.07 & .08 & -.05 & -.03 & -.01 & .15 \\
\hline 7. PCL-5 & - & - & - & - & - & - & - & .04 & .08 & .04 & $.53^{* *}$ & .03 \\
\hline 8. AT - Childhood & - & - & - & - & - & - & - & - & $.41 * *$ & $.78 * *$ & $.31 * *$ & .11 \\
\hline 9. AT - Adolescence & - & - & - & - & - & - & - & - & - & $.71^{* *}$ & .04 & -.01 \\
\hline 10. AT - Adulthood & - & - & - & - & - & - & - & - & - & - & $.28^{*}$ & .08 \\
\hline 11. BDI & - & - & - & - & - & - & - & - & - & - & - & .03 \\
\hline 12. Verbal IQ & - & - & - & - & - & - & - & - & - & - & - & - \\
\hline
\end{tabular}

Note. $\mathrm{N}$-to-P = Negative to positive, $\mathrm{PCL}=\mathrm{PTSD}$ checklist for DSM-IV, $\mathrm{AT}=$ Adversity and traumatic experiences, $\mathrm{BDI}=\mathrm{Beck}$ depression inventory, Verbal

$\mathrm{IQ}=$ verbal intelligence score, ${ }^{*}=p<.05, * *=p<.01$ 
Table 2. Hierarchical regression analyses for negative-to-positive updating for traumatic content.

\begin{tabular}{|c|c|c|c|c|c|c|c|c|c|}
\hline \multirow[b]{2}{*}{ Predictors } & \multicolumn{3}{|c|}{ PCL } & \multicolumn{3}{|c|}{ PCL } & \multicolumn{3}{|c|}{ PCL } \\
\hline & Estimates & $\mathrm{Cl}$ & $p$ & Estimates & $\mathrm{Cl}$ & $p$ & Estimates & $\mathrm{Cl}$ & $p$ \\
\hline (Intercept) & 2.93 & $2.71-3.14$ & $<0.001$ & 3.02 & $2.80-3.25$ & $<0.001$ & 3.02 & $2.80-3.24$ & $<0.001$ \\
\hline Verbal IQ & 0.00 & $-0.01-0.02$ & 0.682 & 0.00 & $-0.01-0.02$ & 0.528 & 0.00 & $-0.01-0.02$ & 0.546 \\
\hline BDI & 0.02 & $0.01-0.03$ & $<0.001$ & 0.02 & $0.01-0.03$ & $<0.001$ & 0.02 & $0.01-0.03$ & $<0.001$ \\
\hline AT-Childhood & -0.01 & $-0.08-0.06$ & 0.811 & -0.02 & $-0.08-0.05$ & 0.654 & -0.03 & $-0.10-0.04$ & 0.452 \\
\hline AT-Adolescence & 0.06 & $-0.00-0.12$ & 0.065 & 0.07 & $0.01-0.13$ & 0.027 & 0.07 & $0.01-0.13$ & 0.026 \\
\hline AT-Adulthood & -0.03 & $-0.06-0.01$ & 0.175 & -0.03 & $-0.06-0.01$ & 0.165 & -0.02 & $-0.06-0.01$ & 0.201 \\
\hline N ACQ - traumatic & -0.05 & $-0.23-0.14$ & 0.617 & -0.09 & $-0.28-0.09$ & 0.306 & -0.10 & $-0.29-0.08$ & 0.261 \\
\hline $\mathrm{N}$-to-P - traumatic & & & & -0.14 & $-0.26--0.02$ & 0.021 & & & \\
\hline N-to-P Cue - traumatic & & & & & & & -0.11 & $-0.20--0.03$ & 0.010 \\
\hline N-to-P Context- traumatic & & & & & & & -0.02 & $-0.11-0.07$ & 0.650 \\
\hline Model test & $F(6,73)=$ & $6.23, p<.001$ & & $F(7,72)=$ & $6.47, p<.001$ & & $F(8,71)=$ & $5.98, p<.001$ & \\
\hline$R^{2} / R^{2}$ adjusted & $0.339 / 0$ & .284 & & $0.386 / 0$ & .326 & & $0.403 / 0$ & .335 & \\
\hline
\end{tabular}

Note. $\mathrm{BDI}=$ Beck depression inventory, Verbal IQ = verbal intelligence score, $\mathrm{N}=$ Negative, $\mathrm{ACQ}=\mathrm{Acquisition}, \mathrm{N}$-to- $\mathrm{P}=\mathrm{Negative}$ to positive, $\mathrm{AT}=$ Adversity and traumatic experiences. 
Table 3. Hierarchical regression analyses for negative-to-positive updating for neutral content.

\begin{tabular}{lcccccc}
\hline & \multicolumn{3}{c}{ PCL } & \multicolumn{5}{c}{ PCL } \\
\multicolumn{1}{c}{ Predictors } & Estimates & $C l$ & $p$ & Estimates & $C l$ & $p$ \\
\hline (Intercept) & 2.89 & $2.67-3.10$ & $<0.001$ & 2.97 & $2.73-3.20$ & $<0.001$ \\
Verbal IQ & 0.00 & $-0.01-0.02$ & 0.670 & 0.00 & $-0.01-0.02$ & 0.604 \\
BDI & 0.02 & $0.02-0.03$ & $<0.001$ & 0.02 & $0.02-0.03$ & $<0.001$ \\
AT-Childhood & -0.01 & $-0.08-0.06$ & 0.849 & -0.01 & $-0.08-0.06$ & 0.805 \\
AT-Adolescence & 0.06 & $0.00-0.13$ & 0.048 & 0.07 & $0.00-0.13$ & 0.039 \\
AT-Adulthood & -0.03 & $-0.07-0.01$ & 0.139 & -0.03 & $-0.06-0.01$ & 0.152 \\
N ACQ - neutral & -0.00 & $-0.20-0.19$ & 0.986 & -0.03 & $-0.23-0.16$ & 0.731 \\
N-to-P - neutral & & & & -0.10 & $-0.23-0.03$ & 0.133 \\
Model test & \multicolumn{2}{l}{$F(6,73)=6.17, p<.001$} & & $F(7,72)=5.71, p<.001$ & \\
$\mathrm{R}^{2} / \mathrm{R}^{2}$ adjusted & $0.336 / 0.282$ & & $0.357 / 0.294$ & \\
\hline
\end{tabular}

Note. $\mathrm{BDI}=$ Beck depression inventory, Verbal IQ = verbal intelligence score, $\mathrm{N}=$ Negative, $\mathrm{ACQ}=$ Acquisition, $\mathrm{N}$-to- $\mathrm{P}=$ Negative to positive, $\mathrm{AT}=$ Adversity and traumatic experiences . 